\title{
A
}

Acta HealthMedica

Acta HealthMedica (ISSN: 2414-6528)

http://www.ActaHealthMedica.com

Volume: 1, Issue: 2, June 2016, Pages: 23-28, DOI: http://dx.doi.org/10.19082/ah23

\section{HARDINESS AND OCCUPATIONAL BURNOUT AMONG NURSING MANAGERS IN MASHHAD UNIVERSITY OF MEDICAL SCIENCES}

\author{
Zahra Rahim Zadegan ${ }^{1}$, Amirhossein Kohan ${ }^{2}$, Lida Jarahi ${ }^{3}$
}

1: M.S.c, Medical Surgical Nursing. Iranian Social Security Organization, Mashhad, Iran

2: M.A., Educational Administration, Mashhad University of Medicine Science, Mashhad, Iran

3: M.D., Associate Professor of Community Medicine, Department of Community Medicine, Faculty of Medicine, Mashhad University of Medical Sciences, Mashhad, Iran

\section{TYPE OF ARTICLE: ORIGINAL}

\begin{abstract}
Introduction: Exhaustion of energy or burnout is a significant problem for health care and medical staff, especially in nursing and nursing management. Burnout is a syndrome consisting of emotional exhaustion, depersonalization, feeling disillusioned, and helplessness in that individuals typically face increased emotional, mental, and physical stress. This study aimed to investigate job burnout and its relation to hardiness in nursing managers.

Methods: In this cross-sectional study, which was carried out in 13 educational hospitals of Mashhad University of Medical Sciences in 2014 on 237 nursing managers, 96 nursing managers were selected randomly based on a Morgan Table. Data were collected using Maslach Burnout Inventory. SPSS version 20 was applied for analysis, and descriptive statistics, chi-square test, and Pearson correlation coefficients were used.

Results: Participants were 65 females and 31 males with a mean age of 37.5 (7.6) and average work experience of 12.2 (3.5) years. Sixty-three percent of nursing managers had a high level of hardiness, $24 \%$ had a low level of hardiness, and $13 \%$ had an average level of hardiness. From a gender perspective, there was no statistically significant difference in the hardiness levels $(p=0.999)$. Pearson correlation test showed a significant inverse correlation between hardiness and burnout $(\mathrm{r}=-0.52, \mathrm{p}<0.001)$.

Conclusion: Study results showed a medium negative correlation between hardiness and occupational burnout, in that, by reduction of hardiness, burnout increased in nursing managers. Although education of methods for the capability of managing unfavorable conditions in a nursing training program can reduce burnout in the high-stress occupational environment, it is necessary for the policymaker to pay attention to nurses' problems and offer encouragement, advantages, and periods of furlough.
\end{abstract}

KEYWORDS: Hardiness, Burnout, Nursing managers

\section{INTRODUCTION}

As one of the important factors in development or flaring up of disease, especially psychological illness (1), full stress occupational environment and emotional or physical exhausted triggers can cause chronic stress syndrome in personnel (2). Job stress is the result of undesirable interaction between the individual and his or her working environment (3). If the job stress in the occupational environment is more than usual or more than a person's tolerance, it can result in physical and psychological adverse effects and endanger one's health; this ultimately leads to reduced quality of a person's performance (4). One component that has attracted a lot of attention in occupational stress issues is burnout or exhaustion of physical and mental energy due to the job (5). Burnout is a chronic syndrome that includes symptoms such as fatigue from work, and resignation from occupational activities is a psychological reaction to stress (6). Baker et al., in several surveys, have introduced a number of important personality factors associated with burnout, e.g., locus of control, self-confidence, progress motivation, and hardiness (psychological hardiness) (7). Kobbasa et al. were among the first researchers who, among the

\section{Correspondence:}

Associate Professor Dr. Lida Jarahi, Department of Community Medicine, Faculty of Medicine, Mashhad University of Medical Sciences, Mashhad, Iran. Tel: +98.3518829262, Fax: +98.3518829263, E-mail: jarahil@mums.ac.ir Received: March 17, 2016, Accepted: April 15, 2016, Published: June 2016 iThenticate screening: March 19, 2016, English editing: April 21, 2016, Quality control: April 22, 2016 (C) 2016 The Authors. This is an open access article under the terms of the Creative Commons Attribution-NonCommercialNoDerivs License, which permits use and distribution in any medium, provided the original work is properly cited, the use is non-commercial and no modifications or adaptations are made. 
demographic variables of the relationship between stress and disease, took personality traits such as hardiness into consideration (8). Hardiness is a combination of beliefs about ourselves and the world, which consists of three components: commitment, control, and challenge seeking. Hardiness takes root in concepts such as having purpose and meaning in life, worthiness of an enthusiastic and passionate life, power and responsibility out of the individual's authority and freedom, the importance of the individuals' subjective experience, and effective roles in building society, which have placed much emphasis in the theories of personality existentialists $(9,10)$. In other words, burnout occurs from the interaction of many factors; one of the most important includes personality traits such as psychological hardiness. Inexhaustible people may consider stressful events potentially favorable in comparison with exhaustible people. Therefore it is possible to follow-up the actual reason for these events, interpret them in their lives, and learn points from these events that are worthy for their future life (11). Considering that burnout is a major problem in a health care system, and nursing managers, as the most important and the busiest managers in this system, face secondary complications for burnout, for various reasons, including absenteeism, turnover, frequent delays, and reduction of performance quality, investigation of the level of burnout among nursing managers and also the investigation of its related factors can help us to identify a manager's problems. The aim of this cross-sectional study was to evaluate the relationship between hardiness and burnout in nursing managers of educational hospitals of Mashhad University of Medical Sciences, Iran.

\section{MATERIAL AND METHODS}

\subsection{Research design and setting}

This cross-sectional study was carried out on nursing managers of educational hospitals of Mashhad University of Medical Sciences between September 2014 and November 2014. The study population was all nursing executive managers, including matrons, supervisors, and head nurses of wards.

\subsection{Sampling}

The sample size was determined with 96 individuals of 237 nursing managers based on a Morgan Table. Participants were selected by simple random sampling. Researchers prepared a list of all nursing managers of 13 educational hospitals of Mashhad University of Medical Sciences; then, by using the table of random numbers, the required number of samples were selected.

\subsection{Selection criteria}

Nursing managers, employed in the educational hospital of Mashhad University of Medical Sciences, Iran, were included in this study. No exclusion criteria were set for this study.

\subsection{Data collection}

Based on nursing managers' work schedules, we referred to the relevant sections; after explaining the purpose of the research and how to complete the questionnaires and obtaining their satisfaction in regards to if they intend to attend the study, the subjects were asked to complete the questionnaires. The instruments of the study included two questionnaires of the Maslach Burnout Inventory. The burnout questionnaire was given to 50 bachelor students of Ahwaz Islamic Azad University by Najarian et al. in an introductory research (12). In the Najarian research, 10 items were removed, 10 other items were modified, and an introductory 90-point scale was provided for a number of professors in the department of psychology to determine the content validity. Finally, a 90-point scale was developed and implemented. The results of factor analysis showed that, among 90 primary items, 27 items had the highest factor loading; further, to examine the reliability of hardiness sale, the test-retest method was used, and the correlation coefficient between the scores of two tests was $r=0.82$ (12). In this study, to gain reliability of the questionnaire, a test-retest examination was used $(r=0.85)$. Job satisfaction in this study also was examined. In the hardiness questionnaire, based on the mean and standard deviation of the scores, participants in three levels of low hardness score (0-39), average (score 40-60), and high (score 61-81) were examined. In terms of hardiness level in this study, 51 subjects $(51 \%)$ were in the low level of hardiness, $38 \%$ had an average level of hardiness, and $11 \%$ had a high level of hardiness.

\subsection{Research ethics}

Protocol for this study was approved by the Ethics Committee of the Tehran Islamic Azad University Medical Branch, Faculty of Nursing. The participants had to provide an informed consent statement before they were allowed to participate. 


\subsection{Statistical analyses}

SPSS Statistics for Windows (IBM Corp. Released 2011. Version 20.0. Armonk, NY: IBM Corp) was applied for analysis, descriptive statistics, chi-square test, and Pearson correlation coefficients were used. A p-value less than 0.05 was considered as a meaningful level.

\section{RESULTS}

Of 96 participants, 65 persons were females (67\%). The mean age of nursing managers was $37.9 \pm 7.6$ years, and the mean work experience was $12.5 \pm 7.3$ years. Seventy-eight participants $(81.2 \%)$ had a bachelor's degree, 89 (93\%) participants were either a head nurse, supervisor, or head nurse and supervisor at the same time. Table 1 shows the frequency of demographic variables and level of job satisfaction in participants. Of the nursing managers, 61 (63\%) have burnout at a high level, 24\% at a low level, and 13\% had burnout at an average level. Table 1 shows the frequency of occupational burnout in the dimensions of emotional exhaustion, depersonalization, and lack of personal success in nursing executive managers.

Table 1. Frequency of demographic variables and level of job satisfaction of nursing executive managers in educational hospitals of Mashhad University of Medical Sciences

\begin{tabular}{|c|c|c|c|c|}
\hline Variables & \multicolumn{2}{|l|}{ Subgroups } & $\mathrm{n}$ & $\%$ \\
\hline \multirow[t]{2}{*}{ Gender } & \multicolumn{2}{|l|}{ Male } & 31 & 33 \\
\hline & \multicolumn{2}{|l|}{ Female } & 65 & 67 \\
\hline \multirow[t]{3}{*}{ Age (year) } & \multicolumn{2}{|l|}{$25-35$} & 9 & 9.5 \\
\hline & \multicolumn{2}{|l|}{$35-45$} & 77 & 80.5 \\
\hline & \multicolumn{2}{|l|}{$>45$} & 10 & 10 \\
\hline \multirow[t]{4}{*}{ Marital status } & \multicolumn{2}{|l|}{ Single } & 9 & 9.5 \\
\hline & \multicolumn{2}{|l|}{ Married } & 81 & 84.5 \\
\hline & \multicolumn{2}{|l|}{ Divorced } & 3 & 3 \\
\hline & \multicolumn{2}{|l|}{ Widowed } & 3 & 3 \\
\hline \multirow[t]{3}{*}{ Educational level } & \multicolumn{2}{|l|}{ Bachelor } & 78 & 81.2 \\
\hline & \multicolumn{2}{|l|}{ Master } & 16 & 16.6 \\
\hline & \multicolumn{2}{|l|}{$\mathrm{PhD}$} & 2 & 2.2 \\
\hline \multirow[t]{4}{*}{ Work experience (year) } & \multicolumn{2}{|l|}{$<5$} & 2 & 2.1 \\
\hline & \multicolumn{2}{|l|}{$5-10$} & 18 & 18.7 \\
\hline & \multicolumn{2}{|l|}{$10-15$} & 49 & 51.1 \\
\hline & \multicolumn{2}{|l|}{$>15$} & 27 & 28.1 \\
\hline \multirow[t]{2}{*}{ Housing } & \multicolumn{2}{|l|}{ Personal } & 66 & 68.8 \\
\hline & \multicolumn{2}{|l|}{ Leased } & 30 & 31.2 \\
\hline \multirow[t]{3}{*}{ Family monthly income (millions Toman) } & \multicolumn{2}{|l|}{1 to 2} & 1 & 1 \\
\hline & 2.1 to 3 & & 85 & 85.5 \\
\hline & Over 3.1 & & 10 & 10.5 \\
\hline Organizational position & Matron & & 7 & 7.3 \\
\hline & Supervisor & & 31 & 32.3 \\
\hline & Head nurse & & 46 & 47.9 \\
\hline & Supervisor and head nurse & & 12 & 12.5 \\
\hline Job satisfaction & Very low & & 26 & 27.1 \\
\hline & Low & & 21 & 21.8 \\
\hline & Average & & 25 & 26.1 \\
\hline & High & & 24 & 25.0 \\
\hline Occupational burnout & Lack of personal success & Low & 82 & 85.4 \\
\hline & & Moderate & 11 & 11.4 \\
\hline & & High & 3 & 3.2 \\
\hline & Depersonalization & Low & 70 & 72.9 \\
\hline & & Moderate & 23 & 23.9 \\
\hline & & High & 3 & 3.2 \\
\hline & Emotional exhaustion & Low & 80 & 83.3 \\
\hline & & Moderate & 10 & 10.4 \\
\hline & & High & 6 & 6.2 \\
\hline
\end{tabular}


Examining the participants in terms of hardiness level showed that men's mean score in terms of control and commitment is more than the mean score of hardiness in women; in terms of challenge seeking, the mean score of women was more than men, but there was no significant difference between men in women $(p=0.23,0.12,0.42)$. Table 2 shows the mean scores of hardiness in participants between male and female. Table 3 shows the correlation between hardness with the elements of burnout among participants. It was observed that there was a significant medium negative correlation ( -0.3 to -0.5$)$ between hardiness and depersonalization and emotional exhaustion. It was not shown a significant difference in the relationship of burnout and hardiness among education level, economic factor, and marital status in this study.

Table 2. Comparing of the mean scores of hardiness in nursing executive managers in educational hospitals of Mashhad University of Medical Sciences

\begin{tabular}{|c|c|c|c|c|}
\hline Groups & Subgroups & Mean Score & Standard deviation & $\mathrm{p}$-value \\
\hline \multirow{2}{*}{ Control } & Male & 42.1 & 5.4 & \multirow{2}{*}{0.12} \\
\hline & Female & 87.1 & 8.3 & \\
\hline \multirow{2}{*}{ Commitment } & Male & 12.2 & 7.0 & \multirow{2}{*}{0.23} \\
\hline & Female & 16.9 & 5.6 & \\
\hline \multirow{2}{*}{ Challenge } & Male & 52.1 & 5.0 & \multirow{2}{*}{0.42} \\
\hline & Female & 18.1 & 4.3 & \\
\hline
\end{tabular}

Table 3. Correlation between components of burnout and hardiness in nursing executive managers in educational hospitals of Mashhad University of Medical Sciences

\begin{tabular}{|l|l|l|l|}
\hline \multicolumn{2}{|l|}{ Hardiness } & $\begin{array}{l}\text { Correlation Coefficient } \\
\text { (Spearman's rho) }\end{array}$ & p-value \\
\hline \multirow{3}{*}{ Components of burnout } & Lack of personal success & -0.026 & 0.031 \\
\cline { 2 - 4 } & Depersonalization & -0.435 & 0.042 \\
\cline { 2 - 4 } & Emotional exhaustion & -0.341 & 0.001 \\
\hline
\end{tabular}

\section{DISCUSSION}

In this study, examining the relationship between hardiness and burnout was done among nurse managers of hospitals of Mashhad University of Medical Sciences. The results showed that participants, in terms of intensity and frequency of burnout in emotional exhaustion and depersonalization, were at a high level and in the lack of personal success were at a low level. According to the above results and high scores in emotional exhaustion and depersonalization and low scores in the lack of personal success, the studied subjects were experiencing high burnout. The obtained results are consistent with Qiu Hong Lin et al.'s research (2013) in regards to the relationship between job stress and job burnout in executive managers (1). In Hong Lin's study, nursing managers had a high level of burnout and were more willing to leave the workplace. High job stress and low job satisfaction associated with high burnout, particularly in emotional exhaustion and depersonalization, was seen in nursing managers. Khodabakhsh and Mansouri (2010) concluded in their study that burnout is high among nurses; between male and female nurses, there was a significant difference in terms of emotional exhaustion, and females had more emotional fatigue than men, but there was no significant difference in other dimensions of burnout (13). Also of 388 nurses, $64 \%$ had low job satisfaction, 78\% had high job stress, and 74\% had burnout (13). Sahebzadeh's research (2010) in the field of occupational burnout of nursing managers in Esfahan hospitals demonstrated that most rates of burnout with average and how intensity were, respectively, $56.8 \%$ in head nurses, $54.5 \%$ in supervisors, $45 / 5 \%$ in the subjects who were supervisors and head nurses at the same time, $14.3 \%$ in matrons, and $10 \%$ in managers (10). In that study conducted to examine the relationship between the organisational position and burnout, there was a significant relationship between emotional exhaustion and organisational position, which is consistent with the results of Sahebzadeh's study (10).

The results of the present study demonstrated a significant relationship between the dimensions of hardiness and burnout. Thus there is a significant inverse relationship between the variable of commitment and burnout. There is a significant inverse relationship between hardiness and emotional exhaustion $(\mathrm{r}=0.341)$. This means that the lower the hardness level of the subjects, the more emotional exhaustion they will experience. There also is a significant inverse relationship between hardiness and depersonalization $(\mathrm{r}=0.345)$, and there is no significant relationship between hardiness and lack of personal success $(r=0.260, p<0.999)$. There is a significant inverse relationship between the variable of commitment and burnout $(\mathrm{r}=-0.41)$. There is a significant inverse relationship between the 
variable of challenge seeking and burnout $(\mathrm{r}=-0.334)$, thus the higher the challenge seeking is an individual, the lower the burnout likelihood will be. There is a significant inverse relationship between the variable of control and burnout $(\mathrm{r}=0.346)$, which means that the better a person is in the control, the lower the burnout will be. Aghajani also concluded in his study that there is a significant negative relationship between hardiness and burnout in the nurses; thus subjects at a low hardness level had higher burnout and vice versa. They concluded that nurses at a low level of hardness are more likely to experience burnout. In the results of their study, just as the results of the present study, a negative linear relationship was observed between hardiness and burnout (14). On the other hand, in Shakerinia's study (2010), people who showed lower levels of hardiness gained higher scores in burnout. They also had higher scores, especially in emotional exhaustion. Regardless of the source and the severity of mental stress, the researchers demonstrate that people respond differently when facing these pressures, and $62 \%$ of people with low hardiness level had high burnout scores (9). The result of this study is consistent with Silvia's study in 2014 titled "Hardiness and Burnout Syndrome Among Nursing Students." The results of this study demonstrate that there was a significant relationship between burnout and hardiness $(\mathrm{p}=0.02, \mathrm{r}=-0.52)$. That is, people who have low hardiness experience high burnout and people who have a high score in hardiness had low job burnout (11). This study has some limitation in a causal relationship because this is a cross-section survey with no follow-up. Also, participants were selected from educational hospitals that have a higher workload than other hospitals. In this study, some socioeconomic factors were assessed, but it may be possible that relations of burnout with other factors are assessed in future studies.

\section{CONCLUSIONS}

In this study, a medium negative correlation between hardiness and occupational burnout was shown, in that, by reduction of hardiness, burnout increased in nursing managers. People with higher hardness may assess stressful events potentially favorably and may pursue the cause of these events, make a relationship with coworkers, and try to balance the high-stress conditions. Although education of methods for the ability to manage unfavorable conditions in a nursing training program can reduce burnout in a high-stress occupational environment, it is necessary for policymakers to pay attention to nurses' problems and encourage advantages and periods of furlough.

\section{ACKNOWLEDGMENTS:}

The authors acknowledge the kind assistance of the nursing managers of the Mashhad University of Medical Sciences for their contributions to this study.

\section{CONFLICT OF INTEREST:}

There is no conflict of interest to be declared.

\section{AUTHORS' CONTRIBUTIONS:}

All authors contributed to this project and article equally. All authors read and approved the final manuscript.

\section{REFERENCES:}

1) Lin Q-H, Jiang C-Q, Lam TH. The relationship between occupational stress, burnout, and turnover intention among managerial staff from a Sino-Japanese joint venture in Guangzhou, China. Journal of occupational health. 2013; 55(6): 458-67. doi: 10.1539/joh.12-0287-OA.

2) Iqbal F, Abbasi F. Relationship between emotional intelligenc and job burnout among universities professors. Asian journal of social sciences \& humanities. 2013; 2(2): 219-29.

3) Mostaghni S, Sarvghad S. Relationship of Personality Characteristics and Psychological Hardiness with Job Stress of Nurses of Public Sector Hospitals in Shiraz. Knowledge \& Research in Applied Psychology. 2013; 13(4): 124-32.

4) Myhren H, Ekeberg, Stokland O. Job satisfaction and burnout among intensive care unit nurses and physicians. Critical care research and practice. 2013: 1-6. Article ID 786176. doi: 10.1155/2013/786176.

5) Tizdast T, Abbas Ghorbani M, Bajvar M. Relationship between hardiness and nurses' professional burnout. Holistic Nursing And Midwifery Journal. 2013; 23(2): 1-7.

6) Subramanian S, Vinothkumar M. Hardiness personality, self-esteem and occupational stress among IT professionals. Journal of the Indian Academy of Applied Psychology. 2009; 35: 48-56.

7) Bakker AB, Van Der Zee KI, Lewig KA, Dollard MF. The relationship between the big five personality factors and burnout: A study among volunteer counselors. The Journal of social psychology. 2006; 146(1): 31-50. doi: 10.3200/SOCP.146.1.31-50. PMid: 16480120. 
8) Kobasa SC. Commitment and coping in stress resistance among lawyers. Journal of Personality and social Psychology. 1982; 42(4): 707. doi: 10.1037/0022-3514.42.4.707.

9) Shakerinia I, Mohammadpour M. Relationship between job stress and resiliency with occupational burnout among nurses. Journal of Kermanshah University of Medical Sciences (J Kermanshah Univ Med Sci). 2010; 14(2): 1-10.

10) Sahebzadeh M, Karimi S, Hosseini SM, Akhtar DG, Hosseini S. Job Burnout of nursing Administrators and chief Executive Officers in university Hospitals and Its Relation to their demographic features. Health Information Management. 2011; 4 (1): 637-9.

11) da Silva RM, Goulart CT, Lopes LFD, Serrano PM, Costa ALS, de Azevedo Guido L. Hardy personality and burnout syndrome among nursing students in three Brazilian universities - an analytic study. BMC nursing. 2014; 13(1): 1. doi: 10.1186/1472-6955-13-9. PMid: 24678676. PMCid: PMC3975133.

12) Khodabakhsh MR, Mansuri P. Analysis and comparison between frequency and depth of job-burnout aspects among male and female nurses. Zahedan Journal of Research in Medical Sciences. 2011; 13(4): 402.

13) Kooranian F, Khosravi AR, H. E. The relationship between hardiness/ locus of control and burnout in nurses Journal Of Gonabad University Of Medical Sciences. 2008; 14(1): 58-67. 